\title{
Predicting Perceived Emotions in Music: the Impact of Genre
}

\section{Felipe S. Tanios*, Tiago F Tavares}

\begin{abstract}
In this work, we assessed the impact of using genre information in the automatic classification of perceived emotion in music. In this process, we developed a dataset in which tracks were mapped according to their genre and their perceived emotion. Our results show that using a specific classifier for each genre yields better results than using a single classifier, with no genre information, for Indie-Rock, Jazz, Heavy-Metal, and Classical music. However, classification result were poor for Bossa-Nova. Therefore we speculate that Bossa-Nova conveys emotion differently than the other tested genres.
\end{abstract}

\section{Key words:}

Music genre, automatic classification, emotion

\section{Introduction}

People within a social group are surrounded by similar social constructs. Since emotions are socio-cultural constructs $^{1}$, people in the same social group tend to share similar emotion perceptions.

This work analyzes music as a socio-cultural construct. In this context, music can be seen as a method for communication and social interaction ${ }^{2}$. Furthermore, music genres are often part of the identity of a group that shares common interests ${ }^{3}$.

Usually automatic prediction of perceived emotion can be done by mapping pieces to a content-inspired vector space $^{4}$. This can be adapted to the prediction of emotions ${ }^{5}$.

\section{Results and Discussion}

To test our hypothesis, first we mapped each track from a dataset (comprising 183 tracks) to a 136-dimentional vector according to their texture features. After, we executed a stratified 10-fold cross-validation test using support vector machines for classification. We performed two tests as shown in Image 1. In the first, we used the whole dataset and ignored genre. In the second, we split dataset according to genre and then performed isolated classification processes for each genre.

Image 1. Experiment Flowchart

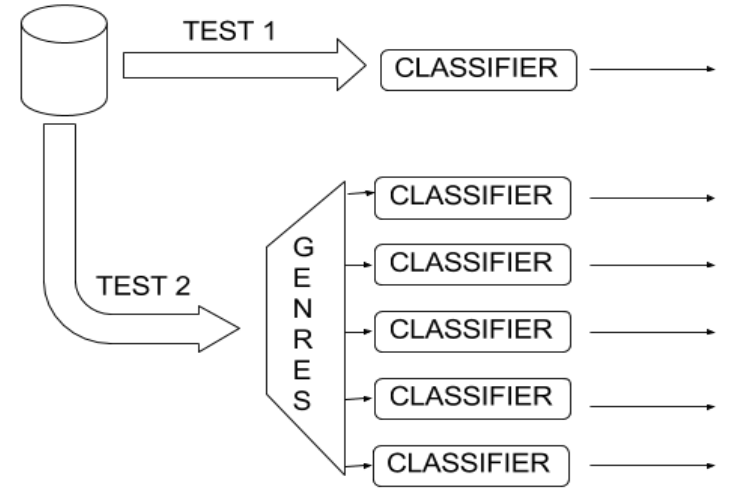

Results, shown in Chart 1, comprise the mean and standard deviation of F1-score. Also we report the results of a t-test between the results obtaned in the first test and the results for each genre individually. It can be seen that the only significant difference $(p<5 \%)$ is related to the Bossa-nova genre. These results suggest that emotions within the Bossa-Nova genre are conveyed in using other means than musical texture.

Chart 1. Mean and standard deviations of F1-scores of each genre individually and all genres together.

\begin{tabular}{|c|c|c|c|}
\hline Test & Genre & F1-Score & P \\
\hline 1 & All & $0.77 \pm 0.10$ & - \\
\hline \multirow{4}{*}{2} & Jazz & $0.85 \pm 0.21$ & 0.26 \\
\cline { 2 - 4 } & Heavy-Metal & $0.79 \pm 0.14$ & 0.11 \\
\cline { 2 - 4 } & Indie-Rock & $0.85 \pm 0.24$ & 0.76 \\
\cline { 2 - 4 } & Classical & $0.86 \pm 0.24$ & 0.25 \\
\cline { 2 - 4 } & Bossa Nova & $0.56 \pm 0.21$ & 0.0005 \\
\hline
\end{tabular}

\section{Conclusions}

The results obtained in this work show that for Jazz, Heavy-Metal, Indie-Rock and Classical music, perceived emotions can be predicted using only texture information. This is not valid for music within the Bossa-Nova genre. This can be related to its typical musical setting, consisted mainly of guitar and voice. Therefore, future word in this field should focus on using other resources such as lyrics to this specific genre.

\section{Acknowledgement}

\footnotetext{
1MAURO GUILHERME PINHEIRO KOURY. Emoções, sociedade e cultura. EDITORA CRV, 2009.

${ }^{2}$ Yi-Hsuan Yang and Homer H. Chen. Prediction of the distribution of preceived music emotions using discrete samples. IEEE Transactions on Audio, Speech and Language Processing, 19(5):2184-2196, 2002.

${ }^{3}$ Beatriz Ilari. Música, comportamento social e relações interpessoais. Psicologia em Estudo, 11(1):191-198, 42006.

${ }^{4}$ George Tzanetakis and Perry Cook. Musical genre classification of audio signals. IEEE Transactions on Speech and Audio Processing, 10(5):293-302, 2002.

${ }^{5}$ Xiao Hu and J. Stephen Downie. Improving mood classification in music digital libraries by combining lyrics and audio. In Proceedings of the 10th Annual Joint Conference on Digital Libraries, JCDL'10, pages 159-168, New York, NY, USA, 2010. ACM.
} 